\title{
MicroRNA- 128-3p Mediates Lenvatinib Resistance of Hepatocellular Carcinoma Cells by Downregulating c-Met
}

\author{
Xin $X u^{1,2}$, Wenjing Jiang ${ }^{\prime}$, Peng Han ', Jingyan Zhang ${ }^{3}$, Liquan Tong $\mathbb{D}^{3}$, Xueying Sun (D) \\ 'Hepatosplenic Surgery Center, The First Affiliated Hospital of Harbin Medical University, Harbin, I5000 I, People's Republic of China; ${ }^{2}$ Department \\ of General Surgery, Daqing Oil Field General Hospital, Daqing, I63000, People's Republic of China; ${ }^{3}$ Department of General Surgery, The Fifth \\ Affiliated Hospital of Harbin Medical University, Daqing, I633I6, People's Republic of China
}

Correspondence: Xueying Sun, Hepatosplenic Surgery Center, The First Affiliated Hospital of Harbin Medical University, Harbin, I5000I, People's Republic of China, Email sunxueying@hrbmu.edu.cn; Liquan Tong, Department of General Surgery, The Fifth Affiliated Hospital of Harbin Medical University, Daqing, I63316, People’s Republic of China, Email tlq777666@I63.com

Objective: Lenvatinib is a first-line multikinase inhibitor for advanced hepatocellular carcinoma (HCC), but resistance to the drug remains a major hurdle for its long-term anti-cancer activity. This resistance is thought to be due to overexpression of c-Met. This study aims to identify potential upstream microRNAs (miRNAs) that regulate c-Met, investigate the underlying mechanisms, and seek potential strategies that may reverse such resistance.

Methods: Lenvatinib-resistant HCC (LR-HCC) cells were established from human HCC Huh7 and SMMC-7721 cells. Assays of cell proliferation, cell cycle distribution, apoptosis, RT-qPCR, Western blot analysis and immunohistochemistry were employed. Potential miRNAs were screened by miRNA-target prediction tools and their regulatory effects were evaluated by luciferase reporter assays. Xenograft tumor models were used to evaluate the therapeutic effects.

Results: LR-HCC cells were refractory to lenvatinib-induced growth inhibition and apoptosis in vitro and in vivo. Sustained exposure of cells to lenvatinib resulted in increased expression and phosphorylation of c-Met, and c-Met inhibition enhanced the effects of lenvatinib in suppressing LR-HCC cells. Among eleven miRNA candidates, miR-128-3p displayed the most vigorous activity to negatively regulate c-Met and was downregulated in LR-HCC cells. MiR-128-3p mimics inhibited proliferation and induced apoptosis of LR-HCC cells, and enhanced the effects of lenvatinib in cell culture and animal models. MiR-128-3p and c-Met participate in the mechanisms underlying lenvatinib resistance through regulating Akt that mediates the apoptotic pathway and ERK (extracellularsignal-regulated kinase) modulating cell cycle progression.

Conclusion: The present results indicate that the miR-128-3p/c-Met axis may be potential therapeutic targets for circumventing lenvatinib resistance in HCC and warrant further investigation.

Keywords: hepatocellular carcinoma, lenvatinib, drug resistance, c-Met, microRNA-128-3p

\section{Introduction}

Hepatocellular carcinoma (HCC) is the third leading cause of cancer-related death worldwide. ${ }^{1}$ HCC is extremely resistant to systemic chemotherapy with conventional cytotoxic drugs, which exhibit limited beneficial effects in HCC patients. ${ }^{2}$ Currently, four tyrosine kinase inhibitors (TKIs) are being used for the clinical management of advanced HCC. Among them, sorafenib is the pioneering drug, while regorafenib and cabozantinib are second-line drugs after the failure of sorafenib. ${ }^{3}$ Lenvatinib has recently been approved as a first-line drug since it achieves similar efficacy to sorafenib in clinical trials, ${ }^{4-6}$ breaking the silence that had plagued the plight of molecular targeted drugs in treating HCC for over 10 years. However, the resistance to lenvatinib minimizes its therapeutic benefits, thus, necessitating an urgent need to further explore its mechanism of action to help identify novel molecular targets. 
Lenvatinib displays its anti-cancer activities by targeting the same molecules as sorafenib, such as vascular endothelial growth factor receptor (VEGFR)-1, 2 and 3, fibroblast growth factor receptors (FGFR) and platelet-derived growth factor receptor (PDGFR), ${ }^{7,8}$ implying that the two drugs may have a similar underlying mechanism for their resistance. The c-Met signaling pathway is activated in HCC tissues and contributes to tumor aggressiveness and poor prognosis. ${ }^{9}$ We have previously reported that the overexpression and activation of c-Met participate in sorafenib resistance of $\mathrm{HCC}$ cells. ${ }^{10} \mathrm{~A}$ recent study suggests that the activation of the hepatocyte growth factor (HGF)/c-Met axis promotes lenvatinib resistance in HCC cells. ${ }^{11}$ However, the upstream mechanisms, and in particular, specific upstream microRNAs (miRNAs/miRs) that regulate c-Met in lenvatinib resistance of HCC cells are yet to be determined.

MiRNAs are a cluster of short non-coding RNAs that are involved in almost all biological behaviors of cells. MiRNAs exert their post-transcriptional regulatory functions mainly by binding to the 3'-untranslated region (3'-UTR) of target genes, leading to degradation or/and prevention of translation of mRNA. ${ }^{12}$ The role of miRNAs has been demonstrated in the progression of many cancers including HCC and some miRNAs have emerged as potential targets for developing novel anti-cancer drugs. ${ }^{12,13}$ A number of miRNAs are involved in drug resistance of HCC through different regulatory mechanisms. ${ }^{14}$ Nevertheless, the identities of upstream miRNAs involved in lenvatinib resistance in HCC are unknown. We, therefore, designed the present study to interrogate and identify potential miRNAs that regulate c-Met and investigate the underlying mechanisms contributing to this resistance.

\section{Materials and Methods}

\section{Cells, Antibodies, and Reagents}

Human HCC SMMC-7721 and Huh7 cells were obtained from Chinese Academy of Sciences Cell Bank (Shanghai, China) and had been tested as negative for mycoplasma infection by using a PCR-based Universal Mycoplasma Detection kit (American Type Culture Collection, Manassas, VA, USA). Cells were cultured in Dulbecco's Modified Eagle Medium (DMEM) (Gibco BRL, Grand Island, NY, USA) supplemented with $10 \%$ fetal bovine serum in a humidified atmosphere of $5 \% \mathrm{CO}_{2}$ at $37^{\circ} \mathrm{C}$. Detailed information regarding antibodies, reagents and kits is described under Supplementary Materials.

\section{Establishment of Lenvatinib-Resistant HCC (LR-HCC) Cells}

The half-maximal inhibitory concentration $\left(\mathrm{IC}_{50}\right)$ of $\mathrm{HCC}$ cells to lenvatinib was initially determined by measuring the viability of cells, which were incubated for $96 \mathrm{~h}$ with serial concentrations of lenvatinib in 96-well plates. Huh7 and SMMC-7721 cells $\left(1 \times 10^{4}\right.$ per well $)$ were then seeded in 6-well plates and incubated with lenvatinib at a starting concentration of $0.25 \mu \mathrm{M}$, which was below their respective $\mathrm{IC}_{50}$. The concentration of lenvatinib was increased by 0.25 $\mu \mathrm{M}$ per week, and two lenvatinib-resistant cell lines (termed SMMC-LR and Huh7-LR, respectively) were obtained 17 weeks later when the concentration of lenvatinib reached $4.5 \mu \mathrm{M}$. The cells were maintained by culturing them in the presence of lenvatinib at $0.5 \mu \mathrm{M}$.

\section{Animal Experiments}

The study had been approved by the Animal Ethics Committee of Harbin Medical University (SYXK20020009) in compliance with the Guideline for Ethical Review of Animal Welfare, China (GB/T 35892-2018). Male BALB/c-nu/nu mice (aging 6-8 weeks) obtained from SLAC Laboratory Animal Co.; Ltd. (Shanghai, China) were housed and received humane care at the Animal Research Center of Harbin Medical University. The establishment of Huh7 and Huh7-LR tumors based on our previous studies ${ }^{10,15}$ is depicted in Supplementary Figure S1. Briefly, cells $\left(5 \times 10^{6}\right)$ were subcutaneously injected into the flake of mice. Since Huh7-LR cells may gradually lose their lenvatinib-resistant behavior in the absence of lenvatinib stimulation, mice injected with Huh7-LR cells were orally administered daily with a low dose of lenvatinib $(2 \mathrm{mg} / \mathrm{kg})$.

\section{Effects of Lenvatinib and Capmatinib}

When Huh7 or Huh7-LR tumors grew to $\sim 100 \mathrm{~mm}^{3}$ in volume 15 or 20 days after cell inoculation, respectively, mice were randomly assigned to treatment groups. Lenvatinib and capmatinib were orally administered daily at a dose of 
$10 \mathrm{mg} / \mathrm{kg}$ and $30 \mathrm{mg} / \mathrm{kg}$, respectively. The doses were selected based on previous reports. ${ }^{10,16}$ Vehicle $(3 \mathrm{mmol} / \mathrm{L} \mathrm{HCl})$ was orally administered and acted as a control. Tumors were measured every 3 days and harvested 15 days after the commencement of treatments.

Effects of miR-I28-3p

When Huh7-LR tumors reached $\sim 100 \mathrm{~mm}^{3}$ in volume, mice were randomly assigned to four groups. Mice in the control and lenvatinib groups received intratumoral injections of negative control oligonucleotides, while mice in the miR-128$3 p$ mimics and lenvatinib + miR-128-3p mimics groups received intratumoral injections of miR-128-3p mimics. Mice in the control and miR-128-3p mimics groups received oral administration of vehicle, while mice in the lenvatinib and lenvatinib + miR-128-3p mimics groups received oral administration of lenvatinib at a dose of $10 \mathrm{mg} / \mathrm{kg}$. Oligonucleotides were dissolved in the injection solution, which was prepared by mixing an equal volume of serumfree medium and Lipofectamine2000. Each tumor received a $50 \mu \mathrm{L}$ injection solution containing $200 \mu \mathrm{g}$ of oligonucleotides on days $0,6,9$, and 12, and was measured every three days. Mice were monitored and euthanized on day 15 .

\section{Cell Proliferation Analysis, Assessment of Cell Cycle and Apoptosis In Vitro, Reverse Transcription-Quantitative Polymerase Chain Reaction (RT-qPCR), Luciferase Reporter Assay, Transfection of Oligonucleotides InVitro, Western Blot Analysis, Immunohistochemistry, In Situ Ki-67 Proliferation Index and In situ Detection of Apoptotic Cells}

Methods for the above procedures have already been described in detail previously ${ }^{10,15,17}$ and are included in the Supplementary Materials.

\section{Statistical Analysis}

GraphPad Prism 8.02 (GraphPad Software, Inc.) was used for statistical analyses. Data are presented as the mean \pm standard deviation. Comparisons were conducted using one-way ANOVA followed by a Tukey's post hoc test. $\mathrm{P}<0.05$ was considered to indicate a statistically significant difference.

\section{Results}

\section{Lenvatinib Induces Overexpression of c-Met in HCC Cells}

LR-HCC cells were shown to be refractory to lenvatinib-induced proliferation inhibition and apoptosis (Supplementary Figure S2), and the lenvatinib-resistant property of Huh-LR cells was also verified in animal models (Supplementary Figure S3). The expression of c-Met mRNA was highly upregulated in SMMC-LR and Huh7-LR cells compared with their respective parental cells (Figure 1A). The expression of c-Met and phosphorylated c-Met (p-Met) proteins showed a similar pattern to c-Met mRNA (Figure 1B). We sequentially investigated the effects of lenvatinib exposure on the c-Met pathway, and discovered that incubation of lenvatinib resulted in an elevated expression of both c-Met and p-Met in either parental or LR-HCC cells (Figure 1C). The expression level of phosphorylated Akt (p-Akt), a major downstream factor of the c-Met pathway, ${ }^{18}$ but not total Akt, was increased by lenvatinib exposure in either parental or LR-HCC cells (Figure 1C). The results were supported by the application of capmatinib, a specific c-Met inhibitor, ${ }^{10,19}$ which showed a stronger inhibitory effect on the proliferation of LR-HCC cells than their respective parental cells, and strengthened the effects of lenvatinib in inhibiting proliferation and promoting apoptosis of LR-HCC cells (Supplementary Figure S4). Capmatinib was also shown to enhance the effects of lenvatinib in inhibiting Huh7-LR tumors in vivo (Supplementary Figure S3C).

\section{Seeking and Identifying Potential miRNAs That Regulate c-Met in LR-HCC Cells}

We screened potential miRNAs that have putative binding sites with the 3'-UTR of human c-Met gene by using multiple miRNA prediction tools including TargetScan (version 7.1; targetscan.org/), miRWalk (version 2.0;), miRTarBase (version 2.0; mirtarbase.mbc.nctu.edu.tw/) and miRanda (version 1.9; omictools.com/miranda-tool), 
A

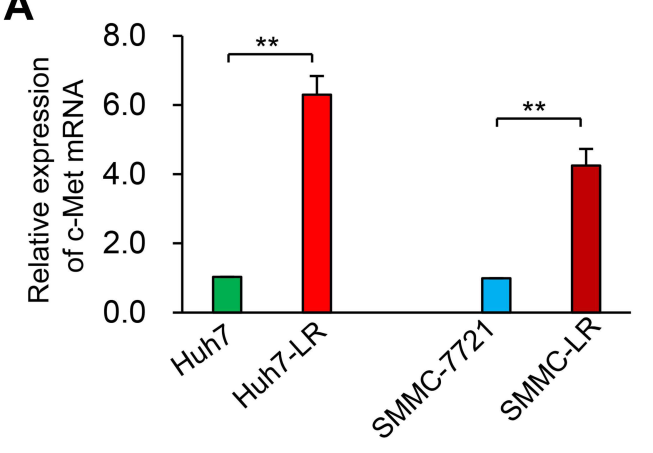

C

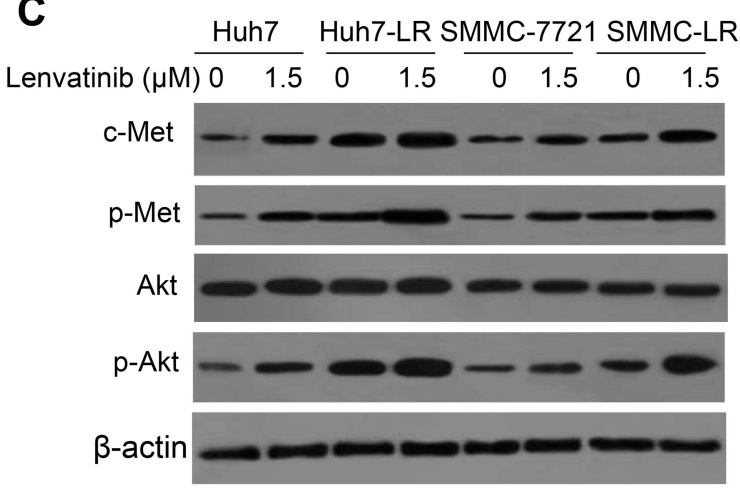

B

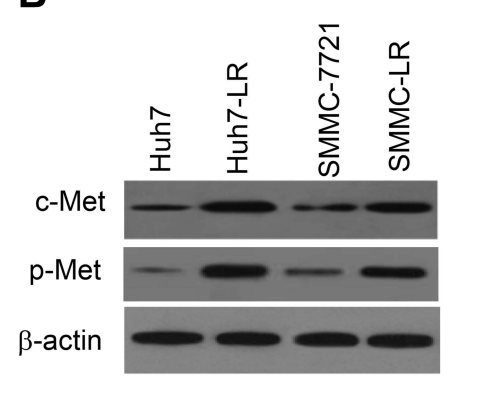

Huh7 $\square$ Huh7-LR

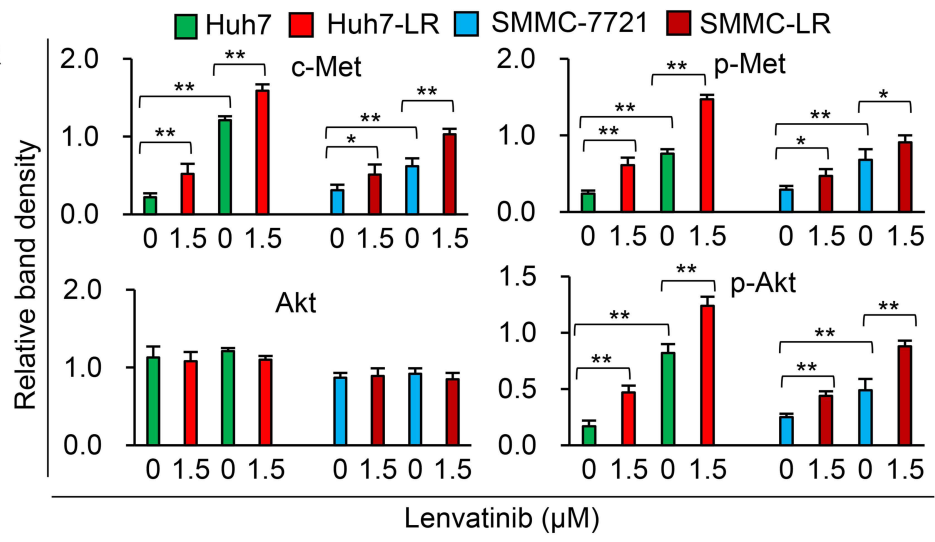

Figure I Lenvatinib exposure induces the activation of the c-Met pathway in HCC cells. (A) The expression of c-Met mRNA in LR-HCC cells, SMMC-LR and Huh7-LR, and their respective parentals, SMMC-772I and Huh7 cells, was measured by RT-qPCR. The level of mRNA from untreated parental cells was defined as I. (B) The expression of c-Met and phosphorylated c-Met (p-Met) protein was measured by Western blot analysis. (C) Cells were incubated in the absence or presence of lenvatinib (I.5 $\mu$ M) for 48 $\mathrm{h}$ and subjected to Western blot analysis. Band densities were normalized to $\beta$-actin. $* \mathrm{P}<0.05 ; * * \mathrm{P}<0.00 \mathrm{I}$.

which excavated 11 miRNA candidates (Supplementary Figure S5). The full-length of 3'-UTR of human c-Met mRNA (Gene ID: 4233) was inserted into an SV40 promoter-driving luciferase reporter vector to create a wild-type vector, based on which a mutated luciferase reporter vector was also constructed by using a mutated 3'-UTR of c-Met gene (Figure 2A). The wild-type vector was co-transfected into Huh7 cells with each miRNA mimic or negative control oligonucleotides (Supplementary Table S1). Among the 11 miRNAs, miR-128-3p displayed the strongest ability to inhibit the luciferase activity in Huh7 cells (Figure 2B). We then detected the expression level of each miRNA in Huh7 and Huh7-LR cells by RT-qPCR with specific primers (Supplementary Table S2). Among the 11 candidates, miR-128-3p was shown to have the largest folds of decreased expression in Huh7-LR compared to Huh7 cells (Figure 2C). The above results indicate that miR-128-3p may be the most potential upstream miRNA that regulates c-Met in HCC cells. A further investigation showed a highly conserved miR-128-3p binding site on the 3'UTR of c-Met in all the available species (Figure 2D). The inhibitory effect of miR-128-3p on the luciferase activity was shown to be dose-dependent (Figure 2E). MiR-128-3p mimics or antagomiR-128-3p significantly altered the luciferase activity in cells co-transfected with the wild-type vector but not the mutated vector (Figure $2 \mathrm{~F}$ ). The results demonstrate that the binding site on the 3'-UTR of c-Met gene is essential for miR-128-3p in mediating its regulatory effect in $\mathrm{HCC}$ cells.

\section{Downregulation of miR-I28-3p by Lenvatinib Contributes to c-Met Overexpression in HCC Cells}

LR-HCC cells expressed significantly lower levels of miR-128-3p than their respective parental cells, and lenvatinib exposure reduced the expression level of miR-128-3p in either parental or LR-HCC cells (Figure 3A). Transfection of miR-128-3p mimics resulted in a significant increase of miR-128-3p expression in LR-HCC cells, while antagomiR-128-3p significantly reduced miR-128-3p expression in parental HCC cells (Figure 3B). On the other 
A

PMIR-IUc-MET-3'UTR

\begin{tabular}{|c|l|}
\hline SV40 Luciferase & MET 3'-UTR \\
\hline
\end{tabular}

\begin{tabular}{|c|c|}
\hline dtype) & (Binding sites, position: 1563-1570) \\
\hline 3'-UTR & $\begin{array}{c}\text { 5'...AACACUGCACUGUGAACAUUU...3' } \\
|||||| \mid\end{array}$ \\
\hline \multicolumn{2}{|c|}{ hsa-miR-128-3p 3'-UUUCUCUGGCCAAGUGACACU-5' } \\
\hline $\begin{array}{l}\text { nutated) } \\
\text { I MET 3'-UTR }\end{array}$ & 5'...AACACUGGUACUGUAACAUUU...3' \\
\hline
\end{tabular}

C

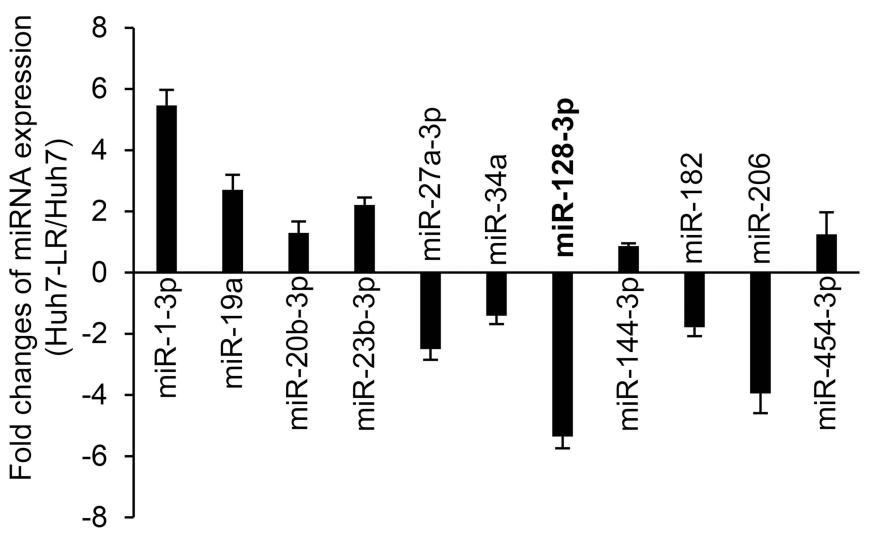

E

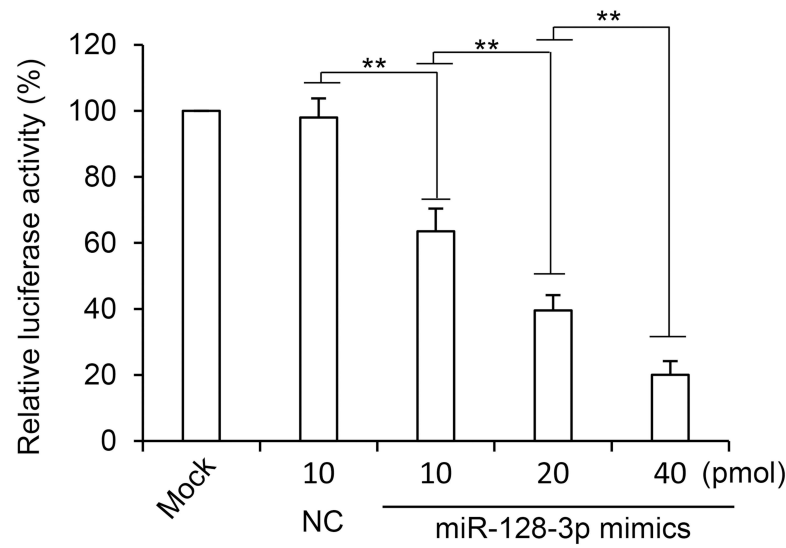

B

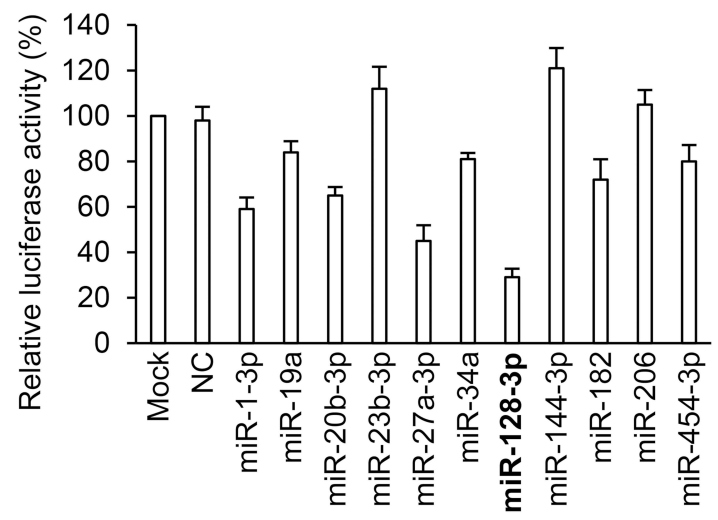

D

Figure 2 MiR-128-3p regulates c-Met by binding to its 3'-UTR. (A) The wild-type (WT) and mutated (MT) luciferase vectors containing the 3'-UTR of human c-Met gene were constructed. (B) Huh7 cells were co-transfected with the WT vector and negative control (NC) or each miRNA mimics as indicated. Mock-treated cells served as controls. (C) The expression level of each miRNA in Huh7 and Huh7-LR was detected by RT-qPCR and their fold changes (Huh7-LR/Huh7) are plotted. (D) The miR-I28-3p binding site on the 3'-UTR of c-Met is highly conserved across all the species. (E) Huh7 cells were co-transfected with the WT vector and miR-I28-3p mimics at different concentrations. (F) Huh7 cells were co-transfected with WT or MT vectors, and NC, miR-I28-3p mimics or antagomiR-I28-3p oligonucleotides. Luciferase activities were measured and normalized to mock-treated cells. $* * \mathrm{P}<0.00 \mathrm{I}$.

hand, transfection of miR-128-3p mimics significantly reduced the expression of c-Met mRNA in LR-HCC cells, while antagomiR-128-3p significantly increased c-Met mRNA expression in parental cells (Figure 3C). The alteration of c-Met protein expression showed a similar trend to the mRNA expression upon transfection of oligonucleotides targeting miR-128-3p (Figure 3D). 
A

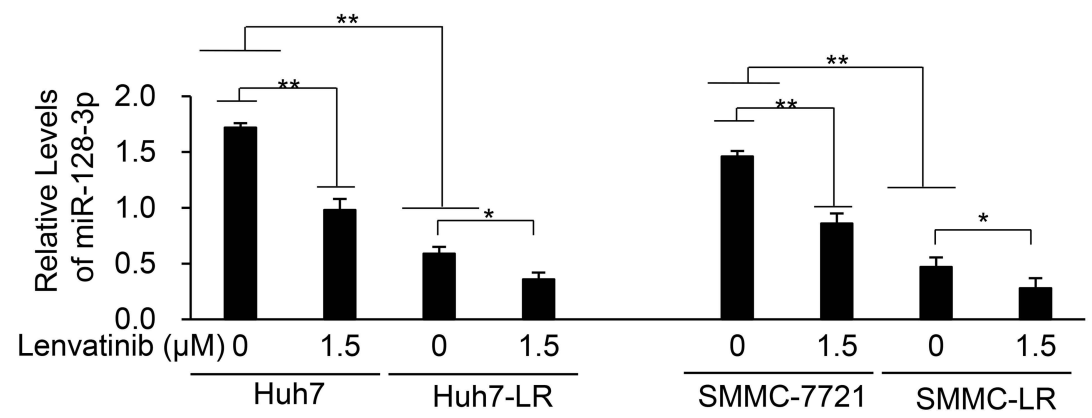

B
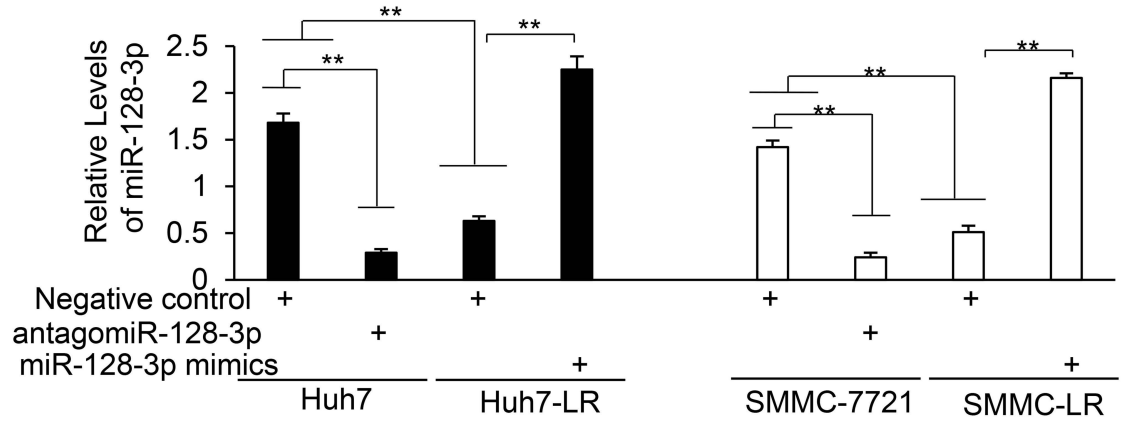

C

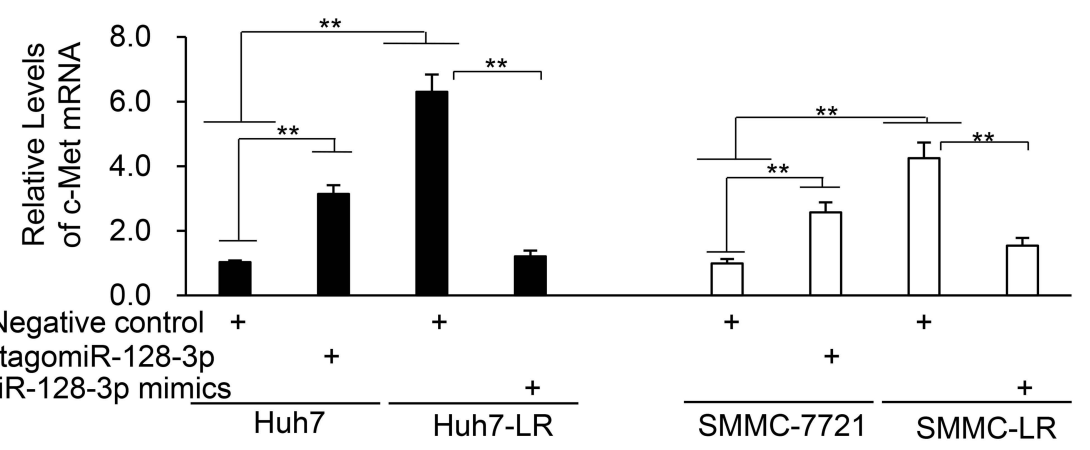

D
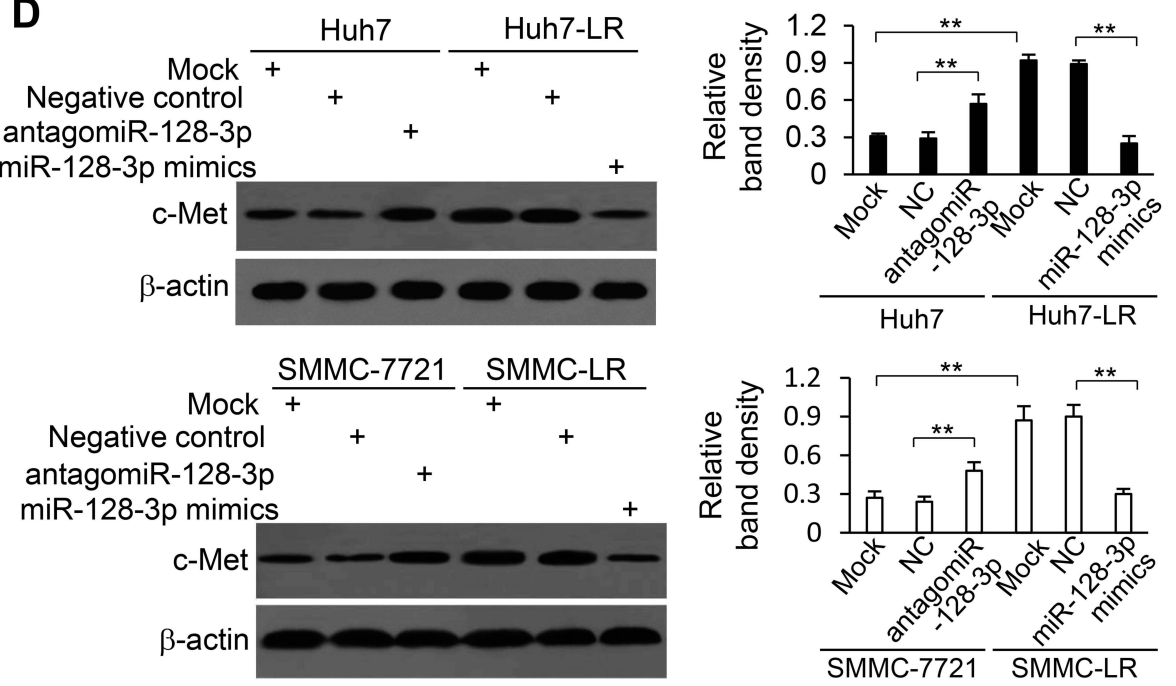

Figure 3 The expression of c-Met is negatively regulated by miR-128-3p in HCC cells. (A) Huh7, Huh7-LR, SMMC-772I and SMMC-LR cells were subjected to qRT-PCR to measure the expression level of miR-128-3p. (B-D) Cells were transfected for $48 \mathrm{~h}$ with negative control (NC), miR-128-3p mimics or antagomiR-I28-3p oligonucleotides. (B and C) Cells were subjected to RT-qPCR to measure the expression level of miR-128-3p (B) and c-Met mRNA (C). (D) Cells were subjected to Western blotting analysis. Band density was normalized to $\beta$-actin. $* \mathrm{P}<0.05$; $* * \mathrm{P}<0.001$. 


\section{MiR-I28-3p Re-Sensitizes LR-HCC Cells to Lenvatinib-Induced Inhibition of Proliferation}

Transfection of miR-128-3p mimics inhibited proliferation of both parental and LR-HCC cells, while antagomiR-128-3p promoted proliferation of parental HCC cells but had little effects on the proliferation of LR-HCC cells (Figure 4A and B). Therefore, we transfected antagomiR-128-3p into parental cells and miR-128-3p mimics into LR-HCC cells, and tested their effects on lenvatinib-induced inhibition of proliferation. AntagomiR-128-3p partially abolished the reduced proliferation of parental HCC cells by lenvatinib (Figure 4C), while miR-128-3p mimics strengthened the effects of lenvatinib in inhibiting proliferation of LR-HCC cells (Figure 4D). The role of miR-128-3p on cell proliferation was supported by its regulatory effects on the ERK (extracellular-signal-regulated kinase)/cyclin D1 pathway through downregulating c-Met expression (Figure 4E and F). Specifically, antagomiR-128-3p transfection upregulated the expression of phosphorylated ERK (p-ERK) and cyclin D1 and partially attenuated the downregulation of these two molecules by lenvatinib in Huh7 cells (Figure 4E). MiR-128-3p mimics downregulated the expression of p-ERK and cyclin D1, and further enhanced the downregulating effects of lenvatinib on these two molecules in Huh7-LR cells

A

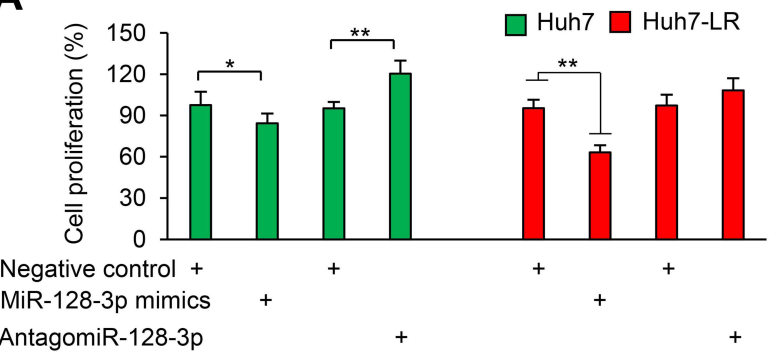

C

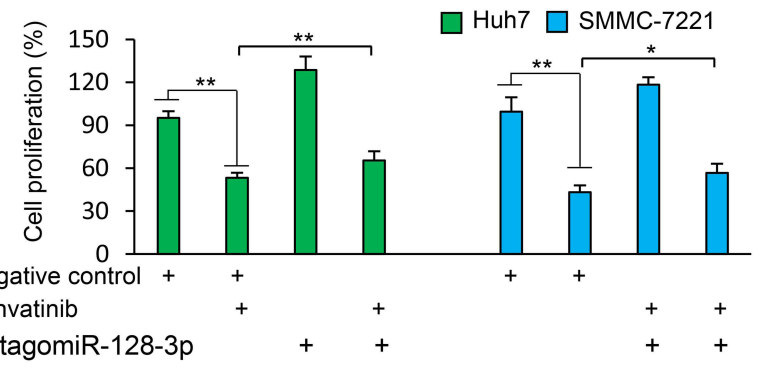

E
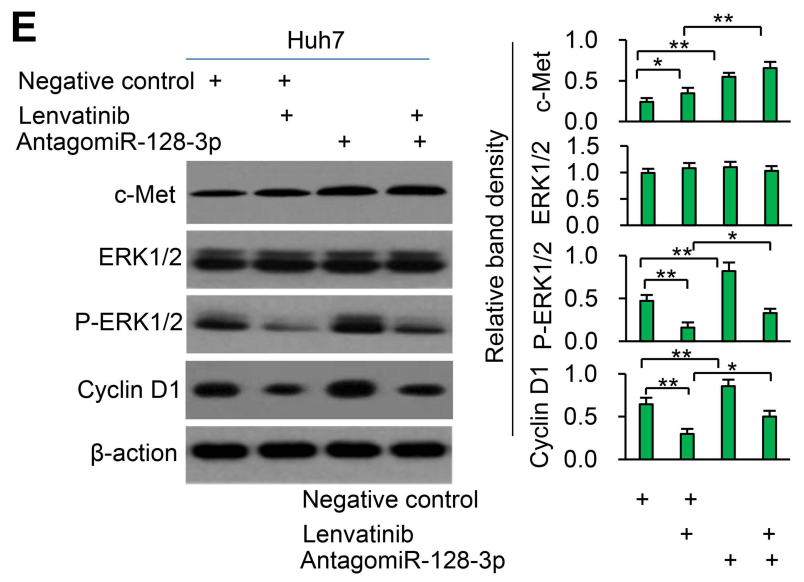

B

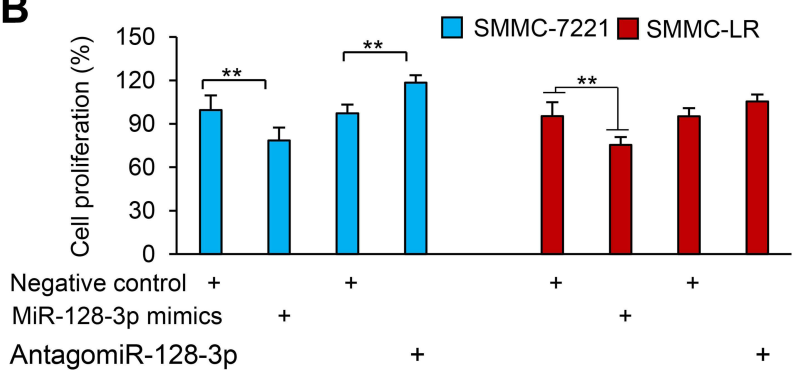

D

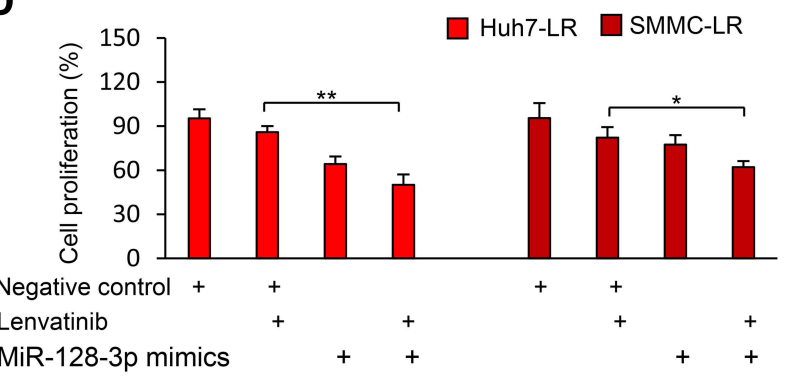

$\mathbf{F}$
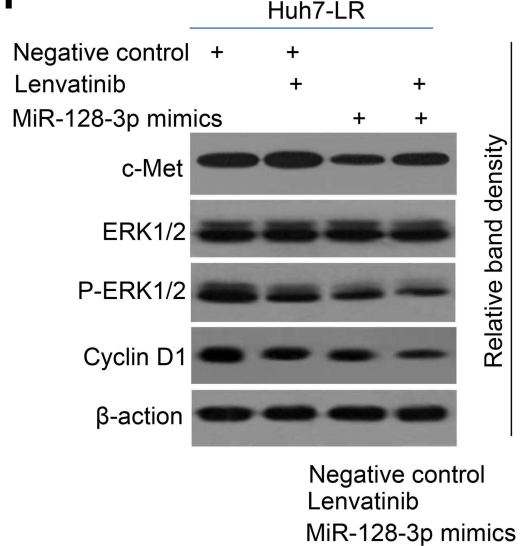

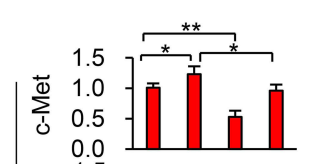

0.0
$\sim 1.5$

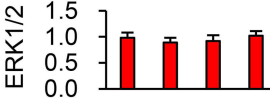

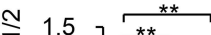

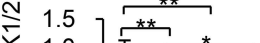

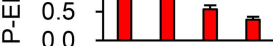

$-1.0{ }^{* *}$

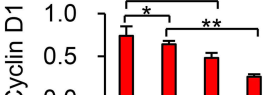

0.0

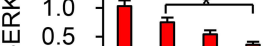

Figure 4 MiR-128-3p mimics re-sensitizes LR-HCC cells to lenvatinib. (A and B) Huh7, Huh7-LR, SMMC-772I and SMMC-LR cells were transfected for 48 h with negative control (NC), miR-128-3p mimics or antagomiR-128-3p oligonucleotides. (C and D) The above cells were incubated in the presence or absence of lenvatinib (0.5 $\mu$ M) for 24 h. (A-D) Cell proliferation (\%) was detected and normalized to mock-treated respective cells. (E and F) Huh7 cells in (C) and Huh7-LR cells in (D) were subjected to Western blot analysis. Band density was normalized to $\beta$-actin. $* \mathrm{P}<0.05 ; * * \mathrm{P}<0.001$. 
(Figure 4F). The altered expression of cyclin D1 in HCC cells was supported by the results of cell cycle distribution (Supplementary Figure S6).

\section{MiR-128-3p Enhances Lenvatinib-Induced Apoptosis in LR-HCC Cells}

Transfection of miR-128-3p mimics induced more apoptosis of both parental and LR-HCC cells, but it induced a higher apoptosis rate of LR-HCC cells than that of respective parental cells; while antagomiR-128-3p had little effect on apoptosis of parental or LR-HCC cells (Figure 5A). We next transfected antagomiR-128-3p into parental cells and miR128-3p mimics into LR-HCC cells, and tested their effects on lenvatinib-induced apoptosis. AntagomiR-128-3p partially abolished the pro-apoptotic effect of lenvatinib on parental cells, while miR-128-3p mimics further increased the proapoptotic activity of lenvatinib in LR-HCC cells (Figure 5B and Supplementary Figure S7). Mechanistically, antagomiR128-3p transfection upregulated the expression of $p$-Akt and phosphorylated glycogen synthase kinase (GSK)-3 $\beta$ (p-GSK-3 $\beta$ ), and partially attenuated the cleavage of caspase- 9 and caspase- 3 induced by lenvatinib in Huh7 cells (Figure 5C). On the other hand, miR-128-3p mimics downregulated the expression of p-Akt and p-GSK-3 3 , and further increased the cleavage of caspase-9 and caspase-3 induced by lenvatinib in Huh7-LR cells (Figure 5D).

A

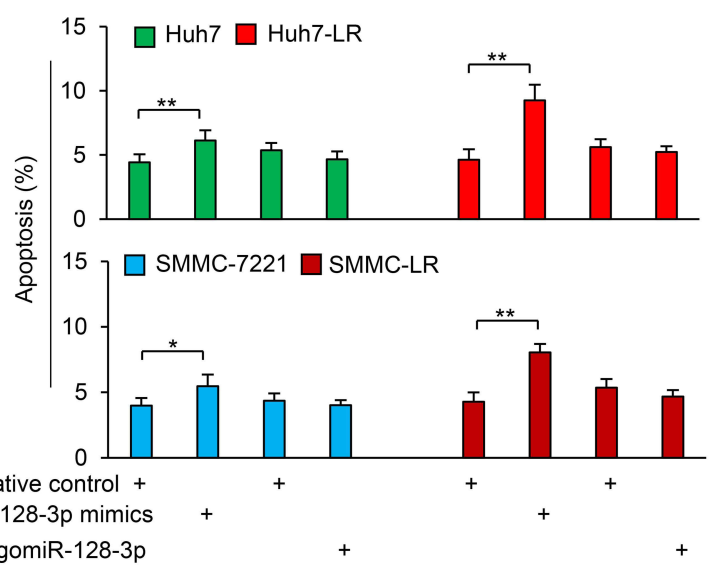

C

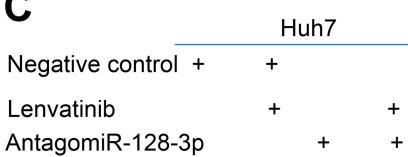

AntagomiR-128-3p
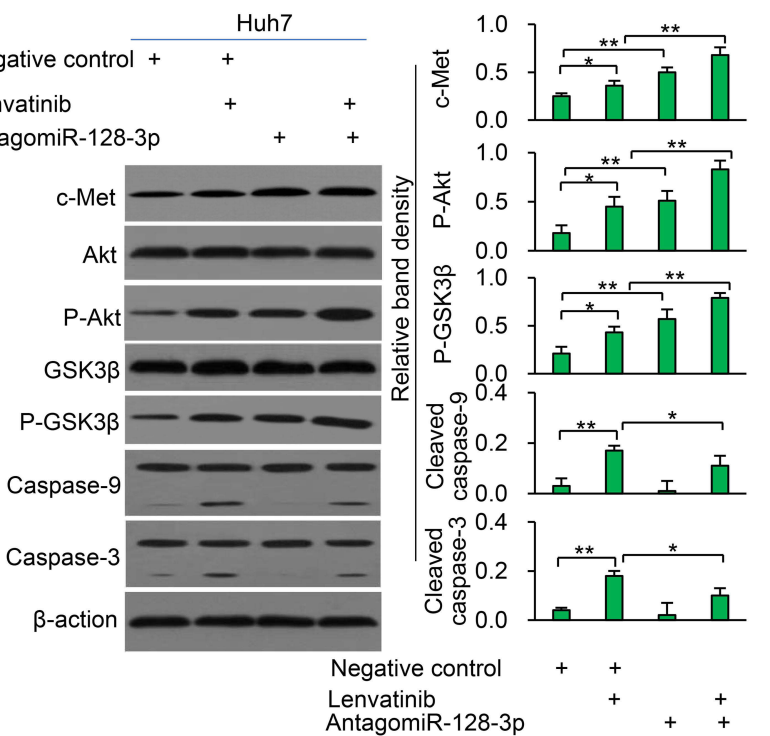

B

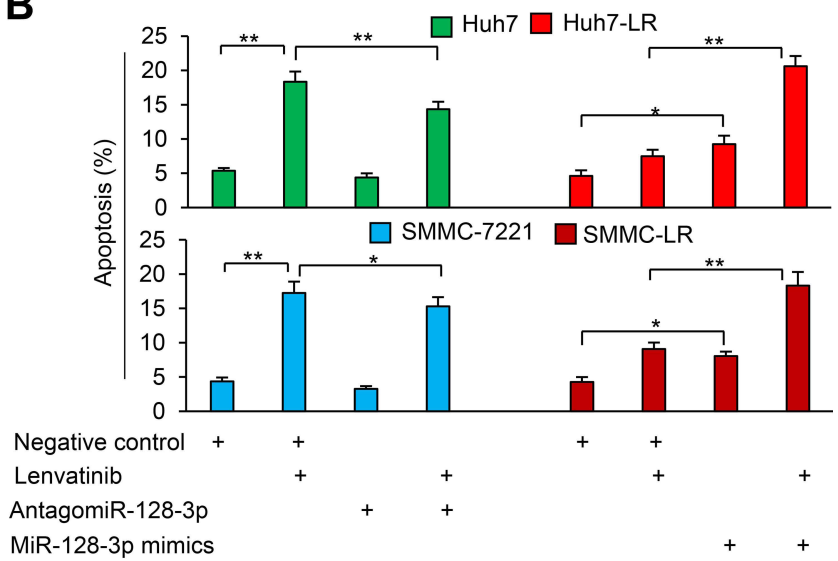

Degative control $+\frac{\text { Huh7-LR }}{+}$

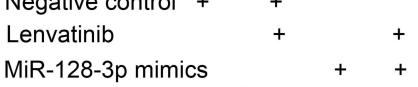

MiR-128-3p mimics
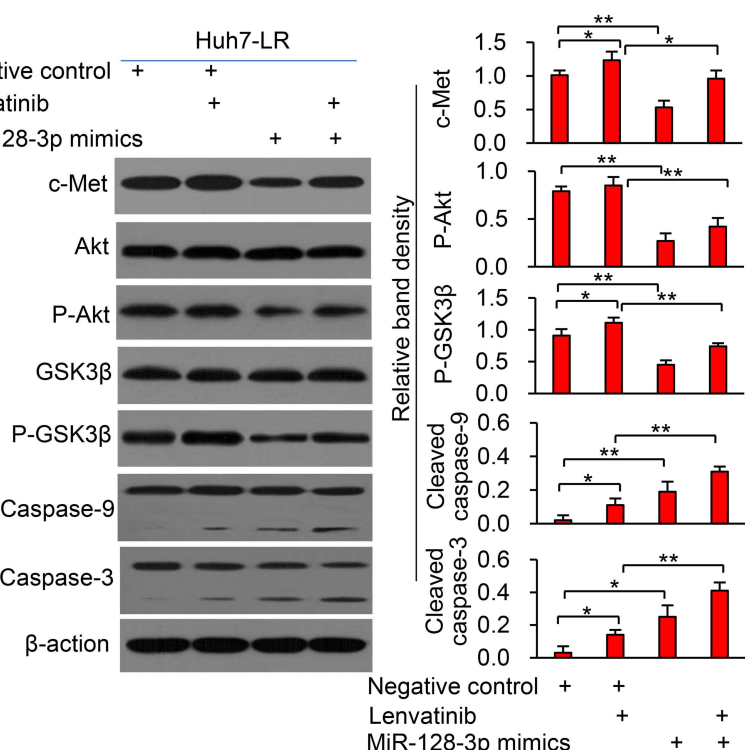

Figure 5 MiR-128-3p enhances lenvatinib-induced apoptosis of HCC cells. (A) Huh7, Huh7-LR, SMMC-772I and SMMC-LR cells were transfected for 48 h with negative control (NC), miR-128-3p mimics or antagomiR-I28-3p oligonucleotides. (B) The above cells were incubated in the presence or absence of lenvatinib (0.5 $\mu \mathrm{M})$ for $24 \mathrm{~h}$. Cell apoptosis (\%) was measured by flow cytometry. (C and D) Huh7 cells (C) and Huh7-LR cells (D) harvested from (B) were subjected to Western blot analysis. Band density was normalized to $\beta$-actin. *P<0.05; **P $<0.001$. 


\section{Transfection of miR-I28-3p Mimics Enhances the Therapeutic Effect of Lenvatinib Against LR-HCC Tumors by Downregulating c-Met Expression In Vivo}

LR-HCC tumors were established in mice, which were assigned to treatment groups (Figure 6A). Treatment of lenvatinib or miR-128-3p mimics resulted in significantly smaller $\left(776.2 \pm 81.5\right.$ or $545.3 \pm 42.7 \mathrm{~mm}^{3}$, respectively) tumors than control tumors $\left(892.4 \pm 63.6 \mathrm{~mm}^{3}\right)$; and the combinational therapy led to even smaller tumors $\left(354.9 \pm 50.5 \mathrm{~mm}^{3}\right)$ than each monotherapy, 15 days after commencement of treatments (Figure 6B). Tumor volume correlated with the weight of tumors (Figure 6C). The downregulation of c-Met in miR-128-3p mimics-treated tumors was confirmed by immunohistochemistry (Figure 6D and E). Treatments of lenvatinib and miR-128-3p mimics significantly increased cellular apoptosis and reduced proliferation in situ compared with control tumors; and the combinational therapy led to even higher apoptosis and lower proliferation indexes than each monotherapy (Figure 6D, F and G). In agreement with the in vitro results (Figure 5D), immunohistochemical analysis of tumor sections demonstrated that miR-128-3p mimics reduced the expression of p-Akt and p-GSK-3 $\beta$, and further increased the expression of cleaved caspase-9 and cleaved caspase-3 induced by lenvatinib in Huh7-LR tumors (Supplementary Figure S8).

\section{Discussion}

HCC patients have a poor prognosis because most of them forgo the opportunity of curative treatments at the time of diagnosis coupled with the notorious resistance to conventional chemotherapy. Although four TKIs have been approved for the treatment of HCC, they show either limited beneficial effects or even an absence of efficacy. ${ }^{3}$ Lenvatinib is a recently approved first-line multi-kinase inhibitor that shows similar therapeutic effects to sorafenib in prolonging the overall survival of patients with advanced HCC. ${ }^{4,5,20}$ However, some patients initially show favorable responses but tumors restart progression after a period of treatment, greatly minimizing its therapeutic effects. ${ }^{4-6}$ The mechanisms underlying acquired resistance to lenvatinib remain unclear.

The overexpression of c-Met is found in more than $80 \%$ of HCC tissues and is associated with a poor prognosis. ${ }^{9,21}$ More importantly, the activation of c-Met is commonly involved in the mechanisms underlying resistance to chemotherapeutic drugs, in particular, molecular targeted therapeutics and anti-angiogenic drugs. ${ }^{21-24}$ Cabozantinib, a second-line approved drug for patients with sorafenib-pretreated advanced HCC, has been shown to exert its therapeutic effects partially by inhibiting c-Met activity. ${ }^{25}$ In agreement, the present study demonstrated that c-Met was markedly upregulated in LR-HCC cells, and lenvatinib exposure increased the expression and phosphorylation of c-Met, and inhibition of c-Met by capmatinib demonstrated a stronger inhibitory effect and enhanced the effect of lenvatinib on LR-HCC cells in vitro and in vivo. Capmatinib is a highly potent and selective c-Met inhibitor, has been approved for the treatment of lung cancer ${ }^{26}$ and has been trialed for treating advanced $\mathrm{HCC} .{ }^{27}$ In support of the present results, the activation of c-Met has been shown to promote lenvatinib resistance in HCC cells. ${ }^{11}$ Another multi-targeted kinase inhibitor sorafenib, which shares the same targets with lenvatinib, has also been shown to activate c-Met signaling. ${ }^{10}$ However, the upstream miRNAs that regulate c-Met in lenvatinib resistance of HCC have not been investigated. To our knowledge, the present study may be the first in revealing a regulatory link between miR-128$3 p$ and $c-M e t$ and in elucidating some of the functional roles of the miR-128-3p/c-Met axis in acquired resistance to lenvatinib in HCC.

MiR-128-3p is regarded as a potential oncomiR as its dysregulation is associated with carcinogenesis, malignant transformation, and metastasis. More precisely, miR-128 is an anti-oncomiR or suppressor oncomiR in HCC as its overexpression suppresses the proliferation and metastasis of HCC cells and positively correlates with the prognosis of HCC patients. ${ }^{28,29}$ Studies have also shown that miR-128-3p increases the chemosensitivity of oxaliplatin-resistant colorectal cancer, ${ }^{30}$ confers chemoresistance-associated metastasis in lung cancer, ${ }^{31}$ enhances the sensitivity of temozolomine in glioma, ${ }^{32}$ and sensitizes HCC cells to sorafenib-induced apoptosis ${ }^{33}$ through regulating various targets. The present study demonstrates that miR-128-3p is downregulated in LR-HCC cells, and miR-128-3p mimics enhances the sensitivity of LR-HCC cells to lenvatinib by downregulating the expression of c-Met, further supporting the involvement of miR-128-3p in chemoresistance though it targets different genes from the above studies possibly because the role of miRNAs is complex, and cell- and disease-dependent. ${ }^{34}$ 
A

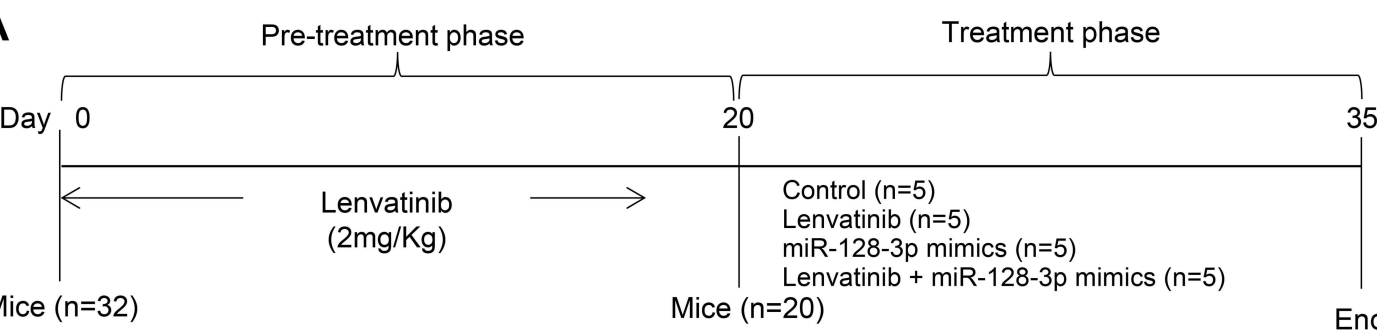

Mice $(n=32)$

Mice $(n=20)$

End

B

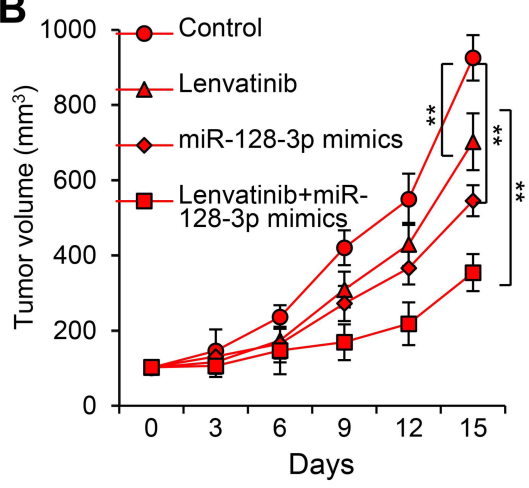

D

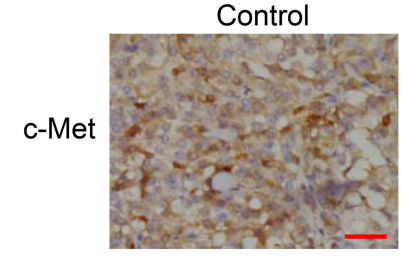

TUNEL

Ki-67

$\mathbf{E}$

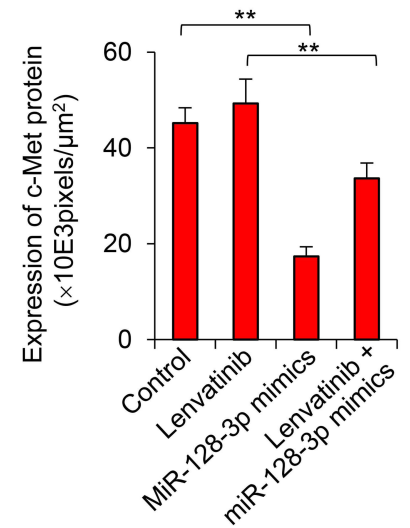

C

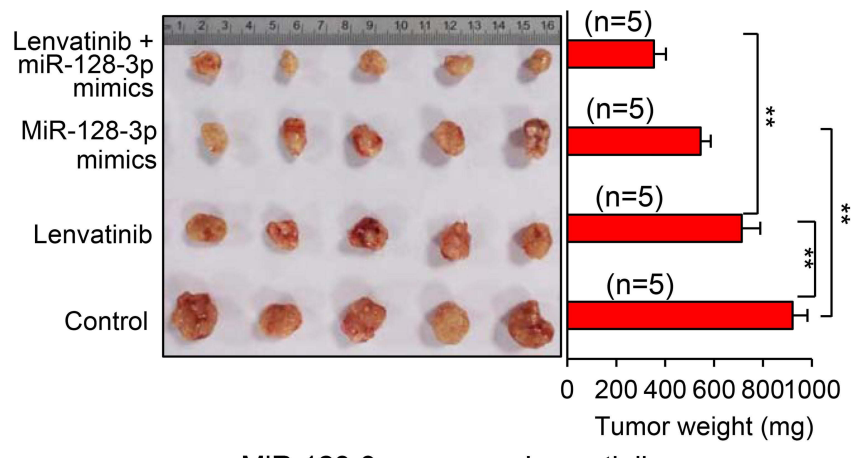

Lenvatinib
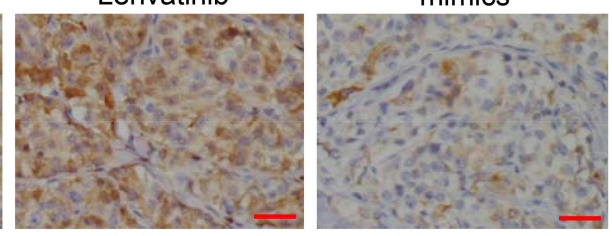

miR-128-3p mimics
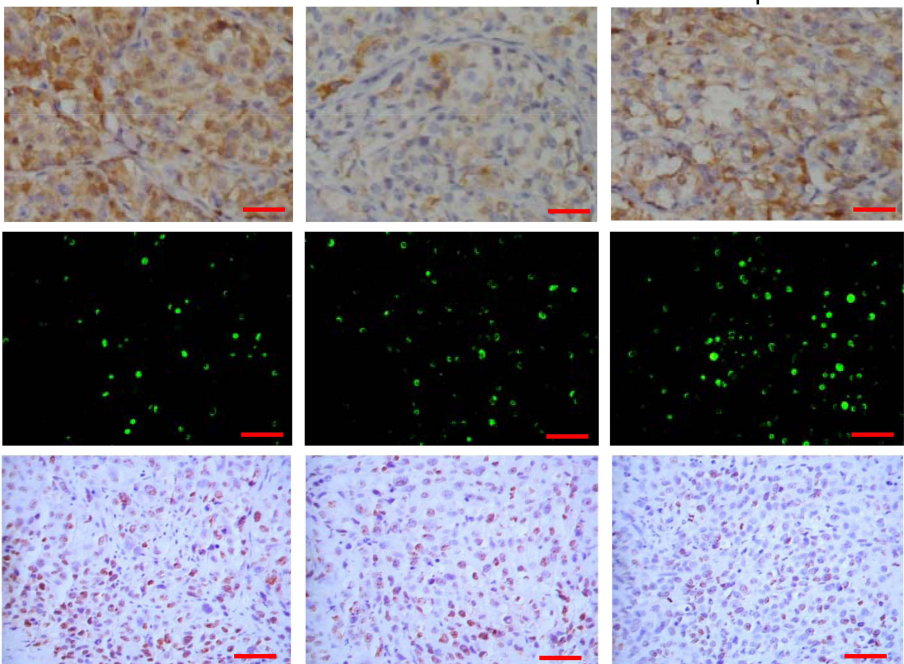

$\mathbf{F}$
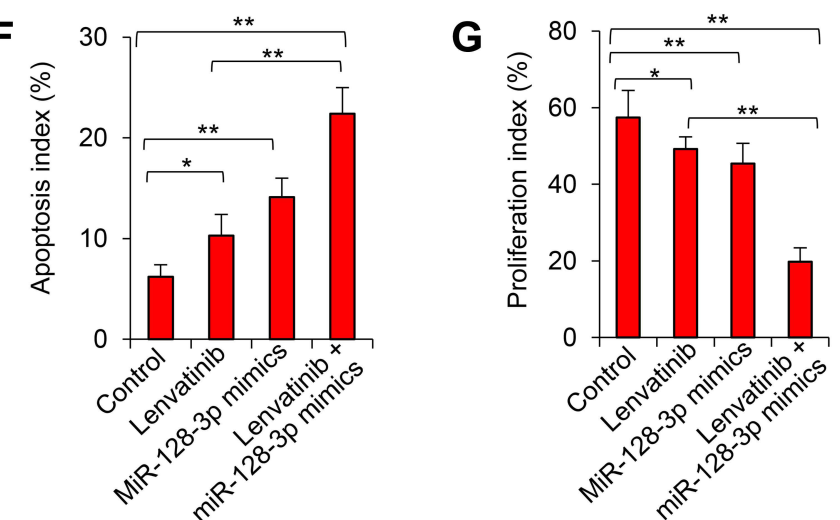

Figure 6 MiR-128-3p mimics enhances therapeutic effects of lenvatinib against LR-HCC tumors in vivo. (A) Huh7-LR tumors were established in mice that were assigned to treatment groups. (B) The size $\left(\mathrm{mm}^{3}\right)$ of tumors was recorded. (C) Tumors harvested at the end of experiments were weighed and photographed. (D) Illustrated are representative tumor sections stained with an anti-c-Met Ab (upper panel), TUNEL (middle panel) or an anti-Ki67 Ab (lower panel). Magnification bar $=100 \mu m$. (E) The expression of c-Met was expressed as pixels $/ \mathrm{um}^{2}$. The apoptosis index $(\mathbf{F})$ and proliferation index $(\mathbf{G})$ were quantified. "n" indicates the number of samples. $* \mathrm{P}<0.05$; $* * P<0.001$. 
Since miRNAs are known to play regulatory roles in a wide variety of diseases, they have been regarded as one of the essential biopharmaceuticals and maybe the next-generation medicine in the future. Many miRNA-based drugs have been developed, in the form of either miRNA mimics or inhibitors; and several miRNA molecules are being evaluated in clinical trials for treating different types of cancer. ${ }^{35}$ Although more studies are necessary, our findings suggest that miR128-3p may be a potential target for developing miRNA therapeutics, which could be used to enhance the efficacy of lenvatinib for treating advanced HCC. One of the weaknesses of the present study is that c-Met overexpression or miR128-3p downregulation has not been validated in clinical specimens, and the therapeutic effects of c-Met inhibition or miR-128-3p overexpression have not been evaluated in patient-derived xenografts, since human LR-HCC tissues are currently not available to us. According to the established guidelines, such as the American Association for the Study of Liver Diseases (AASLD), ${ }^{36}$ lenvatinib is administered to patients with advanced HCC. However, patients with advanced HCC, particularly, those with cirrhosis, benefit little from tissue collection by the invasive procedures, such as laparotomy or laparoscopy or fine-needle biopsy, and face risky complications. In view of stringent human ethical requirements, acquiring informed consents can be difficult or even unwarranted. Postmortem could be another possible way, but the quality of such tissues can be variable and suboptimal for interrogation. However, further validation by using such tissues from patients should be investigated in the future since it will increase the clinical relevance and translation of the present results.

The mechanisms underlying resistance to lenvatinib in HCC remain unclear and highly complex, similar to sorafenib, although the latter has been more widely and deeply investigated. ${ }^{37}$ For instance, the major neuronal isoforms of retrovirus-associated DNA sequences protein (RAS)/rapidly accelerated fibrosarcoma protein (RAF)/mitogen-activated and extracellular-signal-regulated kinase (MEK) pathways may play a critical and central role in HCC escaping from TKIs, and HCC patients with a BRAF mutation are more resistant to TKIs. ${ }^{38}$ Therefore, more mechanistic studies are required to understand drug resistance and seek potential strategies, such as combinational therapies involving alternative pathways, ${ }^{38}$ so that the efficacy of lenvatinib in the treatment of HCC could be improved. In a recent clinical trial, patients with advanced HCC who were unresponsive to lenvatinib treatment showed meaningful responses to the combination of lenvatinib and gefitinib. ${ }^{39}$

The proposed mechanisms depicting how miR-128-3p participates in lenvatinib resistance in HCC by targeting c-Met, the related signaling pathways and the interventions in the present study are depicted schematically in Figure 7. Lenvatinib inhibits ERK and other tyrosine kinase receptors including VEGFR, PDGFR and c-Kit. ${ }^{7,8}$ Exposure of HCC cells to lenvatinib results in a reduction in the expression of miR-128-3p, which negatively regulates the expression of c-Met by binding to the 3'-UTR, thus lenvatinib exposure leads to overexpression of c-Met, providing a plausible rationale that miR-128-3p could enhance the therapeutic effects of lenvatinib in suppressing lenvatinib-resistant HCC cells. Sustained lenvatinib treatment also induces the phosphorylation of c-Met, which in turn activates its downstream factors, Akt and ERK. ${ }^{18}$ Akt activation can increase the phosphorylation of GSK3 $\beta$, thus inhibiting its function, ${ }^{15}$ resulting in suppressed cleavage of apoptotic proteins caspase-9 and caspase-3. Activated Akt can induce the phosphorylation of ERK, leading to the upregulation of cyclin D1 and the promotion of cell cycle transition, thus increasing cell proliferation. As a specific c-Met inhibitor, ${ }^{10,19}$ capmatinib inhibits the activation of c-Met, thus leading to the suppression of the ERK and Akt pathways ${ }^{18}$ and sequential downregulation of downstream factors controlling cell apoptosis and cell cycle arrest.

\section{Conclusions}

In summary, lenvatinib is the second first-line TKI approved for treating advanced HCC, but lenvatinib-treated patients have a median survival time of only 13.6 months. ${ }^{4,5}$ Therefore, investigating plausible mechanisms underlying the resistance and seeking novel therapeutic strategies to promote the efficacy of lenvatinib remain urgent issues in the campaign against the third leading cause of cancer-related death. The present study has demonstrated that the overexpression and activation of c-Met contribute to lenvatinib resistance of HCC cells. In seeking the upstream regulatory mechanisms, we found that miR-128-3p is downregulated in LR-HCC cells, and negatively regulates the expression of c-Met. The miR-128-3p/c-Met axis is involved in lenvatinib resistance by regulating proliferation and apoptosis-related signaling pathways in LR-HCC cells. Either inhibition of c-Met activation or 


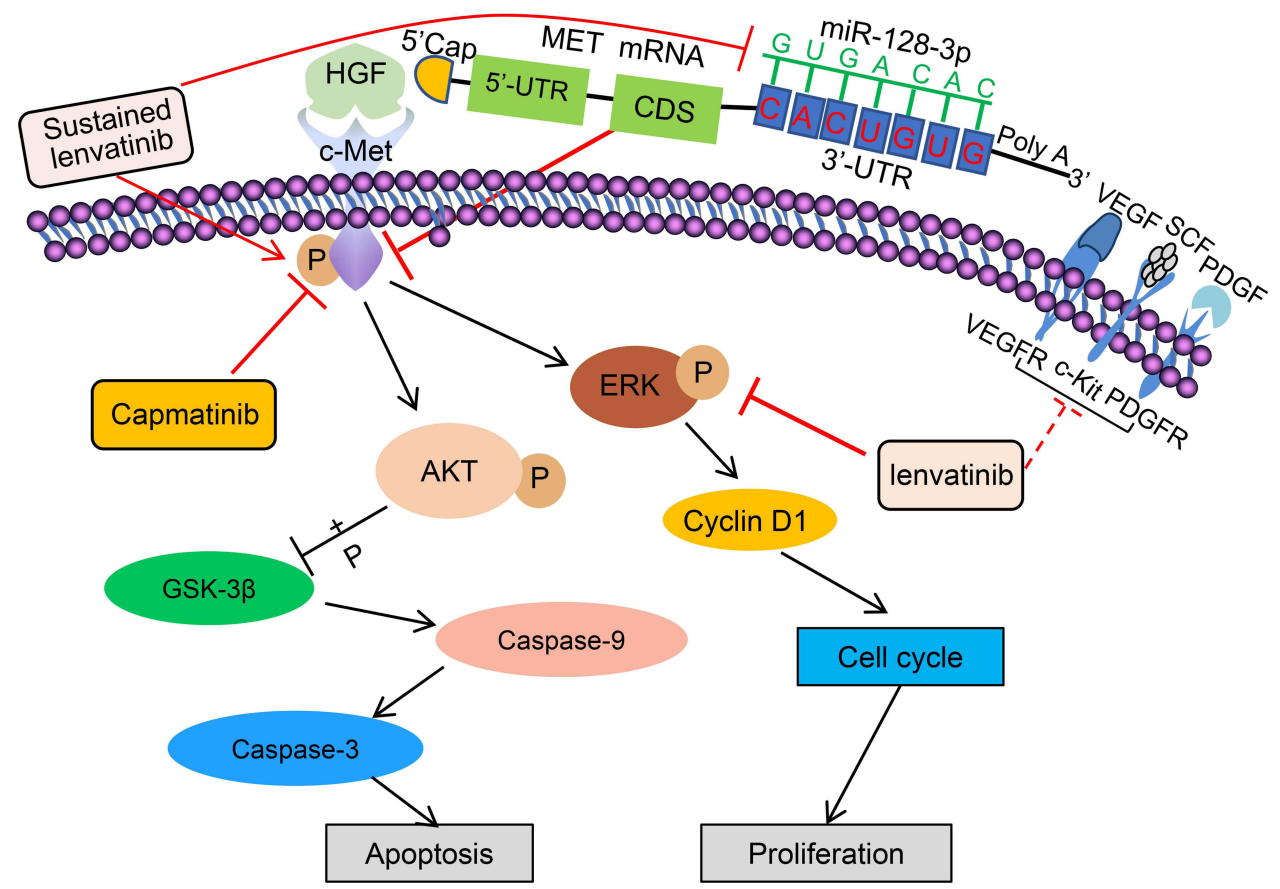

Figure 7 A schematic diagram of proposed mechanisms by which the miR-128-3p/c-Met axis mediates lenvatinib resistance in HCC cells. " $\rightarrow$ " indicates promoting, increasing or activating, while " $\perp$ " indicates inhibiting, reducing or blocking. " $p$ " indicates protein phosphorylation. Solid lines indicate mechanisms examined in the present study, and dotted lines indicate mechanisms reported previously 4,5 .

Abbreviations: ERK, extracellular-signal-regulated kinase; GSK-3 $\beta$, glycogen synthase kinase-3 $\beta$; HGF, hepatocyte growth factor; PDGF, platelet-derived growth factor; PDGFR, platelet-derived growth factor receptor; SCF, stem cell factor; UTR, untranslated region; VEGF, vascular endothelial growth factor; VEGFR, vascular endothelial growth factor receptor.

introduction of miR-128-3p could re-sensitize LR-HCC cells to lenvatinib in vitro and in vivo, suggesting that these molecules may be potential therapeutic targets for overcoming this resistance. Our findings warrant further investigations.

\section{Acknowledgments}

This work was supported in part by grants of National Key Research and Development Program of China (2017YFC1308602), National Natural Scientific Foundation of China (81803010), Heilongjiang Provincial Natural Scientific Foundation (LH2019H006) and an Innovation Fund Project by Heilongiiang Provincial Federation of Trade Unions (2020-12-16). We thank Dr Shiva Reddy from the University of Auckland (New Zealand) for revising and editing the manuscript.

\section{Disclosure}

The authors report no conflicts of interest in this work.

\section{References}

1. Siegel RL, Miller KD, Fuchs HE, Jemal A. Cancer statistics, 2021. CA Cancer J Clin. 2021;71(1):7-33. doi:10.3322/caac.21654

2. Forner A, Reig M, Bruix J. Hepatocellular carcinoma. Lancet. 2018;391(10127):1301-1314. doi:10.1016/s0140-6736(18)30010-2

3. Cheng AL. Pursuing efficacious systemic therapy for hepatocellular carcinoma. Nat Rev Gastroenterol Hepatol. 2021;18(2):95-96. doi:10.1038/ s41575-020-00405-1

4. Thomas H. Liver cancer: lenvatinib non-inferior to sorafenib for hepatocellular carcinoma. Nat Rev Gastroenterol Hepatol. 2018;15(4):190. doi:10.1038/nrgastro.2018.20

5. Kudo M, Finn RS, Qin S, et al. Lenvatinib versus sorafenib in first-line treatment of patients with unresectable hepatocellular carcinoma: a randomised phase 3 non-inferiority trial. Lancet. 2018;391(10126):1163-1173. doi:10.1016/s0140-6736(18)30207-1

6. Vogel A, Qin S, Kudo M, et al. Lenvatinib versus sorafenib for first-line treatment of unresectable hepatocellular carcinoma: patient-reported outcomes from a randomised, open-label, non-inferiority, phase 3 trial. Lancet Gastroenterol Hepatol. 2021;6(8):649-658. doi:10.1016/s24681253(21)00110-2 
7. Ikeda M, Okusaka T, Mitsunaga S, et al. Safety and pharmacokinetics of lenvatinib in patients with advanced hepatocellular carcinoma. Clin Cancer Res. 2016;22(6):1385-1394. doi:10.1158/1078-0432.Ccr-15-1354

8. Oikonomopoulos G, Aravind P, Sarker D. Lenvatinib: a potential breakthrough in advanced hepatocellular carcinoma? Future Oncol. 2016;12 (4):465-476. doi:10.2217/fon.15.341

9. Giordano S, Columbano A. Met as a therapeutic target in HCC: facts and hopes. J Hepatol. 2014;60(2):442-452. doi:10.1016/j.jhep.2013.09.009

10. Han P, Li H, Jiang X, et al. Dual inhibition of Akt and c-Met as a second-line therapy following acquired resistance to sorafenib in hepatocellular carcinoma cells. Mol Oncol. 2017;11(3):320-334. doi:10.1002/1878-0261.12039

11. Fu R, Jiang S, Li J, Chen H, Zhang X. Activation of the HGF/c-MET axis promotes lenvatinib resistance in hepatocellular carcinoma cells with high c-MET expression. Med Oncol. 2020;37(4):24. doi:10.1007/s12032-020-01350-4

12. Wang X, He Y, Mackowiak B, Gao B. MicroRNAs as regulators, biomarkers and therapeutic targets in liver diseases. Gut. 2021;70(4):784-795. doi:10.1136/gutjnl-2020-322526

13. Di Leva G, Garofalo M, Croce CM. MicroRNAs in cancer. Annu Rev Pathol. 2014;9:287-314. doi:10.1146/annurev-pathol-012513-104715

14. Liang Y, Liang Q, Qiao L, Xiao F. MicroRNAs modulate drug resistance-related mechanisms in hepatocellular carcinoma. Front Oncol. 2020;10:920. doi:10.3389/fonc. 2020.00920

15. Zhai B, Hu F, Jiang X, et al. Inhibition of Akt reverses the acquired resistance to sorafenib by switching protective autophagy to autophagic cell death in hepatocellular carcinoma. Mol Cancer Ther. 2014;13(6):1589-1598. doi:10.1158/1535-7163.mct-13-1043

16. Matsuki M, Hoshi T, Yamamoto Y, et al. Lenvatinib inhibits angiogenesis and tumor fibroblast growth factor signaling pathways in human hepatocellular carcinoma models. Cancer Med. 2018;7(6):2641-2653. doi:10.1002/cam4.1517

17. Li W, Dong X, He C, et al. LncRNA SNHG1 contributes to sorafenib resistance by activating the Akt pathway and is positively regulated by miR-21 in hepatocellular carcinoma cells. J Exp Clin Cancer Res. 2019;38(1):183. doi:10.1186/s13046-019-1177-0

18. Peters S, Adjei AA. MET: a promising anticancer therapeutic target. Nat Rev Clin Oncol. 2012;9(6):314-326. doi:10.1038/nrclinonc.2012.71

19. Bouattour M, Raymond E, Qin S, et al. Recent developments of c-Met as a therapeutic target in hepatocellular carcinoma. Hepatology. 2018;67 (3):1132-1149. doi:10.1002/hep.29496

20. Personeni N, Pressiani T, Rimassa L. Lenvatinib for the treatment of unresectable hepatocellular carcinoma: evidence to date. $J$ Hepatocell Carcinoma. 2019;6:31-39. doi:10.2147/jhc.s168953

21. Firtina Karagonlar Z, Koc D, Iscan E, Erdal E, Atabey N. Elevated hepatocyte growth factor expression as an autocrine c-Met activation mechanism in acquired resistance to sorafenib in hepatocellular carcinoma cells. Cancer Sci. 2016;107(4):407-416. doi:10.1111/cas.12891

22. Huang M, Liu T, Ma P, et al. c-Met-mediated endothelial plasticity drives aberrant vascularization and chemoresistance in glioblastoma. $J$ Clin Invest. 2016;126(5):1801-1814. doi:10.1172/jci84876

23. Phan LM, Fuentes-Mattei E, Wu W, et al. Hepatocyte growth factor/cMET pathway activation enhances cancer hallmarks in adrenocortical carcinoma. Cancer Res. 2015;75(19):4131-4142. doi:10.1158/0008-5472.CAN-14-3707

24. Wilson TR, Fridlyand J, Yan Y, et al. Widespread potential for growth-factor-driven resistance to anticancer kinase inhibitors. Nature. 2012;487 (7408):505-509. doi:10.1038/nature11249

25. Shang R, Song X, Wang P, et al. Cabozantinib-based combination therapy for the treatment of hepatocellular carcinoma. Gut. 2021;70 (9):1746-1757. doi:10.1136/gutjnl-2020-320716

26. Wolf J, Seto T, Han JY, et al. Capmatinib in MET exon 14-mutated or MET-amplified non-small-cell lung cancer. $N$ Engl J Med. 2020;383 (10):944-957. doi:10.1056/NEJMoa2002787

27. Qin S, Chan SL, Sukeepaisarnjaroen W, et al. A Phase II study of the efficacy and safety of the MET inhibitor capmatinib (INC280) in patients with advanced hepatocellular carcinoma. Ther Adv Med Oncol. 2019;11:1758835919889001. doi:10.1177/1758835919889001

28. Huang CY, Huang XP, Zhu JY, et al. miR-128-3p suppresses hepatocellular carcinoma proliferation by regulating PIK3R1 and is correlated with the prognosis of HCC patients. Oncol Rep. 2015;33(6):2889-2898. doi:10.3892/or.2015.3936

29. Shi Y, Yan F, Wang F, Pan L. MiR-128-3p suppresses tumor proliferation and metastasis via targeting CDC6 in hepatocellular carcinoma cells. Tissue Cell. 2021;72:101534. doi:10.1016/j.tice.2021.101534

30. Liu T, Zhang X, Du L, et al. Exosome-transmitted miR-128-3p increase chemosensitivity of oxaliplatin-resistant colorectal cancer. Mol Cancer. 2019;18(1):43. doi:10.1186/s12943-019-0981-7

31. Cai J, Fang L, Huang Y, et al. Simultaneous overactivation of Wnt/ $\beta$-catenin and TGF $\beta$ signalling by miR-128-3p confers chemoresistanceassociated metastasis in NSCLC. Nat Commun. 2017;8:15870. doi:10.1038/ncomms15870

32. Zhao C, Guo R, Guan F, et al. MicroRNA-128-3p enhances the chemosensitivity of temozolomide in glioblastoma by targeting c-Met and EMT. Sci Rep. 2020;10(1):9471. doi:10.1038/s41598-020-65331-3

33. Guo XL, Wang HB, Yong JK, Zhong J, Li QH. MiR-128-3p overexpression sensitizes hepatocellular carcinoma cells to sorafenib induced apoptosis through regulating DJ-1. Eur Rev Med Pharmacol Sci. 2018;22(20):6667-6677. doi:10.26355/eurrev_201810_16143

34. Rupaimoole R, Slack FJ. MicroRNA therapeutics: towards a new era for the management of cancer and other diseases. Nat Rev Drug Discov. 2017;16(3):203-222. doi:10.1038/nrd.2016.246

35. Chakraborty C, Sharma AR, Sharma G, Lee SS. Therapeutic advances of miRNAs: a preclinical and clinical update. J Adv Res. 2021;28:127-138. doi:10.1016/j.jare.2020.08.012

36. Bejjani A, Finn RS. The place of novel therapies in the American association for the study of liver diseases guidelines for hepatocellular carcinoma. Clin Liver Dis. 2019;14(2):51-55. doi:10.1002/cld.815

37. Llovet JM, Montal R, Sia D, Finn RS. Molecular therapies and precision medicine for hepatocellular carcinoma. Nat Rev Clin Oncol. 2018;15 (10):599-616. doi:10.1038/s41571-018-0073-4

38. Gnoni A, Licchetta A, Memeo R, et al. Role of BRAF in hepatocellular carcinoma: a rationale for future targeted cancer therapies. Medicina. 2019;55(12). doi:10.3390/medicina55120754

39. Jin H, Shi Y, Lv Y, et al. EGFR activation limits the response of liver cancer to lenvatinib. Nature. 2021;595(7869):730-734. doi:10.1038/s41586021-03741-7 


\section{Publish your work in this journal}

The Journal of Hepatocellular Carcinoma is an international, peer-reviewed, open access journal that offers a platform for the dissemination and study of clinical, translational and basic research findings in this rapidly developing field. Development in areas including, but not limited to, epidemiology, vaccination, hepatitis therapy, pathology and molecular tumor classification and prognostication are all considered for publication. The manuscript management system is completely online and includes a very quick and fair peer-review system, which is all easy to use. Visit http://www.dovepress.com/testimonials.php to read real quotes from published authors.

Submit your manuscript here: https://www.dovepress.com/journal-of-hepatocellular-carcinoma-journal 